\title{
A CHECKLIST OF PTINIDAE (COLEOPTERA BOSTRICHOIDEA) OF IRAN
}

\author{
(*) Universitat de Barcelona, Facultat de Biologia, Departament de Biologia Animal, Avda, Diagonal 645, 08028 Barcelona, \\ Spain. E-mail: av.rodama@gmail.com \\ (**) Young Researchers and Elites Club, Science and Research Branch, Islamic Azad University, Tehran, Iran. \\ E-mail: hghahari@yahoo.com \\ ( ${ }^{\circ}$ Correspondence: hghahari@yahoo.com
}

Viñolas A., Ghahari H. - A checklist of Ptinidae (Coleoptera Bostrichoidea) of Iran.

The fauna of Iranian Ptinidae (Coleoptera: Bostrichoidea) is summarized. In total 43 species belonging to 21 genera and 8 subfamilies are listed: Anobiinae ( 7 genera, 10 species), Dorcatominae (1 genus, 1 species), Ernobiinae (3 genera, 4 species), Eucradinae (2 genera, 2 species), Gibbiinae (1 genus, 3 species), Ptilininae (2 genera, 5 species), Ptininae (3 genera, 8 species) and Xyletininae (2 genera, 10 species). Four species, Gastrallus pubens Fairmaire, 1875, Priobium carpini Herbst, 1793 (Anobiinae), Hyperisus plumbeum (Illiger, 1801) (Ernobiinae), and Stagetus pilula (Aubé, 1861) (Dorcatominae) are newly recorded from Iran.

KeY Words: Coleoptera, Bostrichoidea, Ptinidae, checklist, new record, Iran.

\section{INTRODUCTION}

Worldwide about 220 genera and some 2200 species of Ptinidae are known, and they are distributed throughout the major regions of the world, although they are especially abundant in the driest parts of the subtropical and temperate zones (ArAngo \& Young, 2012). Ptinidae are mainly scavengers, and many species feed equally readily on more or less dried plant or animal materials. Many species are associated with the nests or excrement of other animal species, while others are found in dead or decaying wood, leaf litter or among other plant debris (BELLÉs, 2009; Moseneagu, 2012). Some other species, especially among Anobiinae and Dorcatominae subfamilies, have a very specialized life form. Anobiinae generally are xylophagous, attacking the wood of deciduous and coniferous trees, wild or elaborated (ESPAÑOL, 1992). Dorcatominae species live at the expense of different models of fungi. They can be in fungi on trees and fungi of the rotten wood (VIÑOLAS, $2013 \mathrm{a}, \mathrm{b}, \mathrm{c}, \mathrm{d})$.

Iran forms a large part of the Iranian plateau, and covers an area of $1,623,779 \mathrm{~km}^{2}$. It is bordered to the north by the Caucasus Mountains, Middle Asian natural regions, and the Caspian Sea (-27 m below sea level); to the west by the Anatolian and Mesopotamian regions; to the east by the eastern part of the Iranian plateau (Afghanistan and adjacent west Pakistan) and the Baluch-Sindian region; and finally to the south by the Persian Gulf and Gulf of Oman, which are connected by the latter to the Indian Ocean. Climatologically, Iran is a predominantly arid and semi-arid country, but the northern slopes of the Alburz ranges and the Caspian lowland receive 800 to $2000 \mathrm{~mm}$ annual rainfall, making them the most humid parts of the country. The Dasht-e Kavir and Dasht-e Lut deserts are the driest areas with an annual precipitation of less than $150 \mathrm{~mm}$. The highlands receive between 250 and $800 \mathrm{~mm}$ (ZEHZAD et al., 2002).

This paper lists all the data on Iranian Bostrichidae, and it is a continuation of a series of checklists of Coleoptera of Iran (see Legalov et al., 2010: Curculionoidea; LASOŃ \& GHAHARI, 2013: Kateretidae and Nitidulidae; BunALSKI et al., 2014: Scarabaeoidea; BARTOlozzi et al., 2014: Lucanidae; GHAHARI et al., 2015: Buprestidae; NovÁK \& GHAHARI, 2015: Alleculinae; GHAHARI \& HÁvA, 2015: Silphidae; BEAVER et al., 2016: Platypodinae and Scolytinae; Platia \& GHAHARI, 2016: Elateridae; LiU et al., 2016: Bostrichidae; ZAPPI \& GHAHARI, 2016: Cleridae; THOMAS \& GHAHARI, 2016: Cucujidae, Laemophloeidae, Silvanidae; MirUtenKo \& GHAHARI, 2016: Malachiidae; FANTI \& GHAHARI, 2016: Cantharidae; GHAHARI et al., 2017: Oedemeridae; OTERO et al., 2017: Cryptophagidae).

\section{MATERIAL AND METHODS}

The published data on distribution of the family Ptinidae (Coleoptera) in Iran are summarized by province. Data about nomenclature and distribution data are mainly based on Borowski (2007: Gibbiinae and Ptininae subfamilies) and ZAHRADNíK (2007: Ptinidae family, except Gibbiinae and Ptininae subfamilies), and in other cases the related references are given. When accurate data about local distribution in Iran are lacking in a quoted reference, the mention "Iran (no locality cited)" is used. The provinces of Iran are represented in the Fig. 1.

\section{RESULTS}

This checklist includes 43 Ptinidae species belonging to 21 genera (in brackets the number of species): Anobium Fabricius, 1775 (1), Dignomus Wollaston, 1682 (1), Ernobius Thomson, 1859 (2), Gastrallus Jacquelin du Val, 1860 (3), Gibbium Scopoli, 1777 (3), Hedobia Dejean, 1821 (1), Hemicoelus LeConte, 1861 (2), Hyperisus Mulsant \& Rey, 1863 (1), Lasioderma Stephens, 1835 (5), Nicobium LeConte, 1861 (1), Niptus Boieldieu, 1856 (1), Oligomerm Redtenbacher, 1849 (1), Plumilus White, 1974 (1), Priobium Motschulsky, 1845 (1), Ptilinus Geoffroy, 1762 (4), Ptinomorphus Mulsant \& Rey, 1868 (1), Ptinus Linnaeus, 1767 (6), Stagetus Wollaston, 1861 (1), 


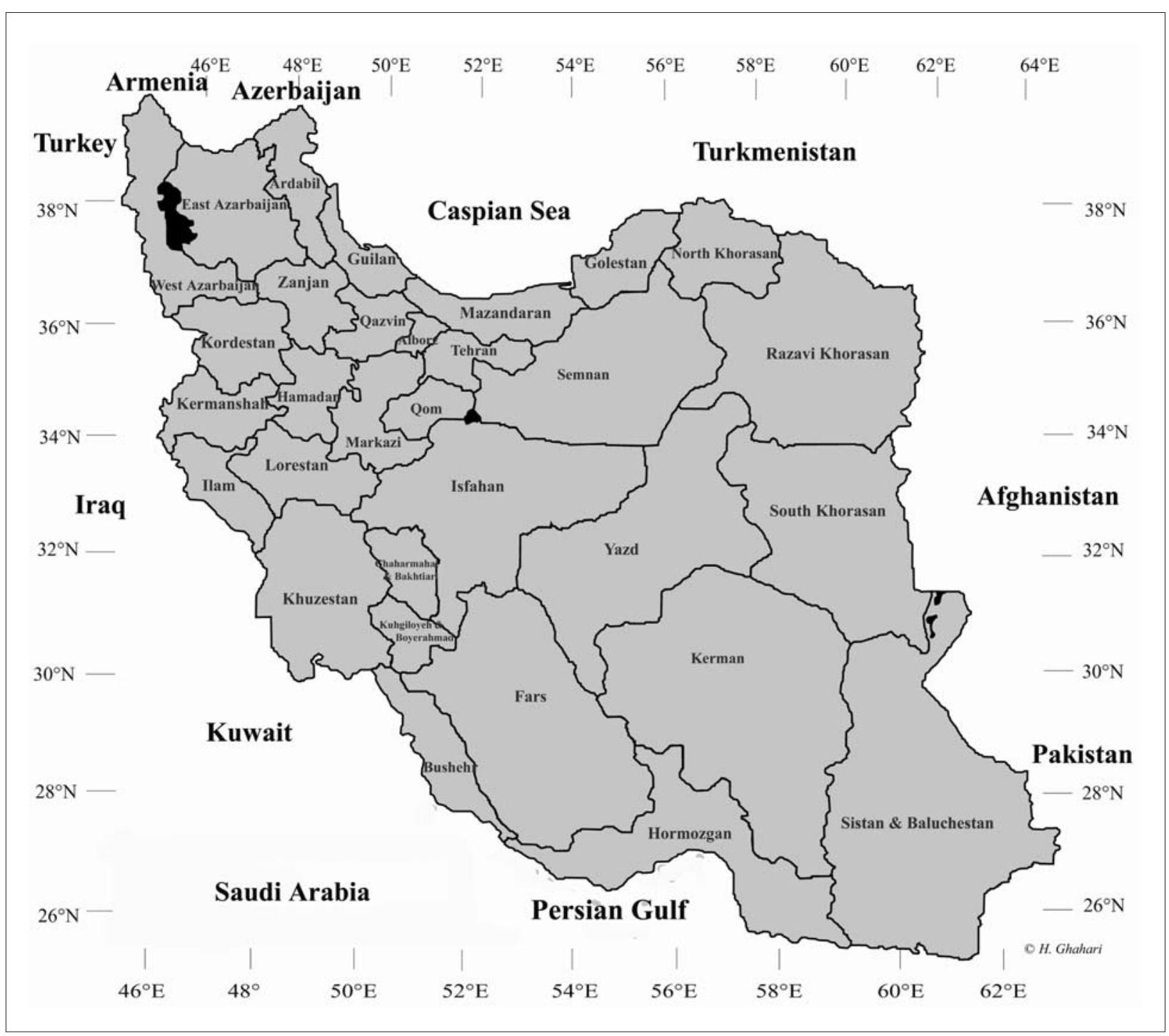

Fig. I - Map of Iran with boundaries of Provinces.

Stegobium Motschulsky, 1860 (1), Xestobium Motschulsky, 1845 (1) and Xyletinus Latreille, 1809 (5). Gastrallus pubens Fairmaire 1875, Hyperisus plumbeum (Illiger, 1801), Priobium carpini Herbst 1793 and Stagetus pilula (Aubé, 1861) are new records for the fauna of Iran.

Family Ptinidae Latreille, 1802

Subfamily Anobiinae Fleming, 1821

Tribe Anobiini Fleming, 1821

Genus Anobium Fabricius, 1775

\section{Anobium punctatum (DeGeer, 1774)}

Byrrhus domesticus Geoffroy, 1785; Anobium striatum A.G. Olivier, 1790 (non Fabricius, 1787); Anobium ruficolle Herbst, 1793; Anobium pubescens Herbst, 1793; Ptinus cylindricus Marsham, 1802; Anobium latreillei Dufour, 1843; Anobium caelatum Mulsant \& Rey, 1864; Hadrobregmus pumilus LeConte, 1865; Anobium amplicolle Broun, 1880; Anobium ruficorne Broun, 1880.

Distribution IN IRAN - Golestan (ABD-RABOU et al., 2005), Guilan, northern and central provinces (MODARRES Awal, 1997), Tehran (Modarres Awal, 1997 as Anobium domesticum (Geoffroy, 1785)), Iran (no locality cited) (ZAHRADNÍK, 2007).

GENERAL DISTRIBUTION - Species currently considered cosmopolitan (ESPAÑOL, 1992; ZAHRADNíK, 2007).

HosTs - Alder, ash, oak, pine, poplar, walnut and other hard wood and coniferous timbers used in construction and furniture (MODARRES AWAL, 1997).

REMARKS - Theocalax formiciformis Westwood, 1832 (Hymenoptera: Pteromalidae) is parasitoid of Anobium punctatum (DeGeer) (ABD-RABOU et al., 2005).

\section{Genus Hemicoelus LeConte, 1861}

Hemicoelus canaliculars (C.G. Thomson, 1863)

DISTRIBUTION IN IRAN - Iran (no locality cited) (ZAHRADNíK, 2007).

General Distribution - Austria, Azerbaijan, Belarus, Belgium, Croatia, Czech Republic, Denmark, Estonia, Finland, France, Germany, Great Britain, Greece, Iran, Italy, Latvia, Malta, Montenegro, Netherlands, Norway, Poland, Romania, Serbia, Slovakia, Spain, Russia, Sweden, Switzerland, Ukraine (ZAHRADNÍK, 2007). 
Hemicoelus fulvicornis (Sturm, 1837)

Anobium morio A. Villa \& G.B. Villa, 1835; Byrrhus ruber Reitter, 1897; Anobium khnzoriani Karapetyan, 1980.

DISTRIBUTION IN IRAN - Iran (no locality cited) (ZAHRADNíK, 2007).

General Distribution - Armenia, Austria, Azerbaijan, Belgium, Bulgaria, Croatia, Czech Republic, Denmark, Finland, France, Georgia, Germany, Great Britain, Greece, Hungary, Italy, Iran, Netherlands, Poland, Romania, Russia, Slovakia, Slovenia, Spain, Sweden, Switzerland, Turkey, Ukraine (ZAHRADNÍK, 2007).

Tribe Gastrallini White, 1982 Genus Gastrallus Jacquelin du Val, 1860

Gastrallus immarginatus (P.W.J. Müller, 1821) Anobium cornicularium Beck, 1817; Anobium exilis Gyllenhal, 1827; Anobium sericatum Laporte, 1840.

DISTRIBUTION IN IRAN - Mazandaran (SAMIN et al., 2016).

General distribution - Armenia, Austria, Belgium, Bulgaria, China, Croatia, Czech Republic, Denmark, France, Germany, Greece, Hungary, Italy, Iran, Japan, Lebanon, Poland, Romania, Slovakia, Spain, Sweden, Switzerland, Ukraine (ZAHRADNÍ,, 2007; SAMIN et al., 2016).

Gastrallus laevigatus (Olivier, 1790)

Anobium parallelum Küster, 1849.

DISTRIBUTION IN IRAN - Isfahan (SAMIN et al., 2015).

General distribution - Algeria, Austria, Belgium, Bosnia-Herzegovina, Bulgaria, Croatia, Cyprus, Czech Republic, France, Georgia, Germany, Greece, Hungary, Iran, Israel, Italy, Japan, Morocco, Netherlands, Poland, Portugal, Russia, Slovakia, Spain, Switzerland, Syria, Tunisia, Turkey, Ukraine (ZAHRADNÍK, 2007; SAMIN et al., 2015).

HOSTS - The species lives in the mistletoe and old trunks of oak, beech, elm, etc. (ESPAÑOL, 1992).

Gastrallus pubens Fairmaire, 1875

Gastrallus striatus Zoufal, 1897; Gastrallus rollei Reitter, 1912; Gastrallus subtuberculatus Pic, 1949.

Examined Material - Golestan province, Minudasht, $37^{\circ} 10^{\prime} \mathrm{N} 55^{\circ} 30^{\prime} \mathrm{E}, 2$ ex., 6.vi.2002, leg. H. Ghahari. New record for Iran.

General Distribution - Azerbaijan, Chad, Egypt, Ethiopia, Israel, Jordan, Kenya, Lebanon, Senegal, Spain, Sudan, Syria, Tunisia, Turkmenistan, Uganda (EsPAÑol, 1963; ZAHRADNÍK, 2007).

HOSTS - Different authors quote records of the species in libraries, where it attacks the old books (ESPAÑOL, 1992).

Tribe Hadrobregmini White, 1982

Genus Priobium Motschulsky, 1845

Priobium carpini (Herbst, 1793)

Anobium serricorne Duftschmid, 1825; Anobium excisum Mannerheim, 1843; Trypopitys dendrobiformis Reitter, 1901.

EXAMINED MATERIAL - West Azarbaijan province, Oshnavieh, $37^{\circ} 03^{\prime} \mathrm{N} 45^{\circ} 05^{\prime} \mathrm{E}, 2$ ex., 14.v.2003, leg. N. Samin. New record for Iran.

GENERAL Distribution - Armenia, Austria, Azerbaijan, Belarus, Belgium, Bosnia-Herzegovina, Bulgaria, Cyprus, Czech Republic, Denmark, Finland, France, Germany, Georgia, Hungary, Italy, Kazakhstan, Latvia, Lebanon, Lithuania, Montenegro, Netherlands, Norway, Poland, Portugal, Romania, Russia (Siberia), Serbia, Slovakia,
Spain, Syria, Sweden, Switzerland, Turkey, Ukraine (ZAHRADNÍ, 2007).

Tribe Nicobiini White, 1982

Genus Nicobium LeConte, 186

Nicobium schneideri Reitter, 1878

DiSTRIBUTION IN IRAN - Iran (no locality cited) (ZAHRADNÍK, 2007).

General Distribution - Azerbaijan, Egypt, Georgia, Iran, Portugal (Azores), Russia,

Turkey, Ukraine (ZAHRADNÍK, 2007).

Tribe Stegobiini White, 1982

Genus Oligomerm Redtenbacher, 1849

Oligomerm ptilinoides (Wollaston, 1854)

Anobium brunneus Duftschmid, 1825 (non A. G. Olivier, 1790); Anobium oculatum Wollaston, 1865; Anobium reyi Ch. Brisout, 1867.

DISTRIBUTION IN IRAN - Iran (no locality cited) (ZAHRADNíK, 2007).

GENERAL DISTRIBUTION - Algeria, Azerbaijan, Austria (Introduced), China, Croatia, Cyprus, Egypt, Francia, Germany (Introduced), Greece, Hungary, Iran, Israel, Italy, Japan, Kazakhstan, Malta, Montenegro, Morocco, Poland (Introduced), Portugal (Madeira Archipelago), Romania, Russia, Serbia, Slovakia, Slovenia, Spain (Canary Islands), Syria, Switzerland, Turkey, Tunisia, Ukraine (ZAHRADNík, 2007).

HosTS - Soft dead wood and wood furniture (ESPAÑOL, 1992).

COMMENTs - Pediculoides ventricosus (Newport, 1855) (Acarina: Pediculoidae) is parasitoid of $O$. ptilinoides (ESPAÑOL, 1992).

Genus Stegobium Motschulsky, 1860

Stegobium paniceum (Linnaeus, 1758)

Anobium ferrugineum Herbst, 1783; Ptinus testaceus Thumberg, 1784; Ptinus upsaliensis Gmelin, 1790; Anobium minutum Fabricius, 1792 (non Fabricius, 1781); Ptinus tenuicornis Marsham, 1802; Ptinus rubellus Marsham, 1802; Anobium tenuestriatum Say, 1825; Anobium ireos A. Villa \& G.B. Villa, 1833; Anobium obesum Melsheimer, 1846; Anobium villosum Melsheimer, 1846; Anobium nanum Küster, 1849; Cis striatopunctata Steinheil, 1873; Cis bonariensis Steinheil, 1873.

DISTRIBUTION IN IRAN - Largely distributed (Modarres Awal 1997), Iran (no locality cited) (ZAHRADNí, 2007).

General Distribution - Cosmopolite (ZAHRADNíK, 2007).

Hosts - All dry plants and animal products and bread, cookies, tobacco, insect collections, wood, paper, seeds, books, grains, legumes and their products and also barley (in granaries (MODARRES AWAL, 1997)).

Subfamily Dorcatominae C.G. Thomson, 1859

Tribe Prothecini White, 1982

Genus Stagetus Wollaston, 1861

Stagetus pilula (Aubé, 1861)

Theca obscurior Pic, 1908; Theca pilula var. rufonotata Pic, 1910.

EXAMINED MATERIAL - Ardabil province, Germi, 39002 
N $47^{\circ} 572$ E, 1 ex., 14.viii.2004, leg. H. Ghahari. New record for Iran.

General distribution - Algeria, Austria, Croatia, Czech Republic, France, Egypt, Germany, Greece, Hungary, Israel, Italy, Kazakhstan, Macedonia, Poland, Slovakia, Slovenia, Spain, Syria, Tajikistan, Turkey, Ukraine (ZAHRADNIK, 2007; ViÑOLAS \& Masó, 2007; VIÑOLAS, 2013b).

\section{Subfamily Ernobiinae Pic, 1912}

Tribe Ernobiini Pic, 1912

Genus Ernobius Thomson, 1859

\section{Ernobius iranicus Zahradnik, 2016}

DiSTRIBUTION IN IRAN - Kermanshah (ZAHRADNIK, 2016).

GENERAL DISTRIBUTION - Endemic to Iran (ZAHRADNIK, 2016).

COMMENTS - ZAHRADNIK (2016) recorded this species from Bachtaraa province (Bisolul). There is not Bachtaraa prov. in Iran (see fig. 1). However, the old name of Kermanshah province is Bakhtaran which we think it is the type locality.

\section{Ernobius mollis mollis (Linnaeus, 1758)}

Anobium testaceus Kugelann, 1792; Anobium sybaris Kugelann, 1792; Anobium convexifrons Melsheimer, 1846; Liozoum sulcatulum Mulsant \& Rey, 1863; Liozoum consimile Mulsant \& Rey, 1863; Liozoum consimile var. laetum Mulsant \& Rey, 1864; Ernobius tarsatus Kraatz, 1881; Ernobius reversus Sharp, 1916.

DisTRIBUTION IN IRAN - Isfahan (GHAHARI et al., 2010), Iran (no locality cited) (MODARRES AWAL, 1997).

General Distribution - Armenia, Austria, Azores, Belarus, Belgium, China, Croatia, Czech Republic, Denmark, Egypt, Estonia, Faeroe Islands, Finland, France, Georgia, Germany, Great Britain, Greece, Hungary, Iceland, Iran, Ireland, Israel, Italy, Japan, Latvia, Lithuania, Macedonia, Morocco, Netherlands, Norway, North Korea, Poland, Portugal (Madeira Archipelago), Romania, Russia, Slovakia, Slovenia, Spain, Sweden, Switzerland, Taiwan, Tunisia, Turkmenistan, Turkey, Ukraine. Introduced in Afrotropical, Australian, Nearctic, Neotropical and Oriental regions (ZAHRADNIK, 2007; GHAHARI et al., 2010).

Hosts - The species lives in conifer stumps (ESPAÑOL, 1992).

Comments - Trychnosoma ernobii Hedqvist 1974 (Hymenoptera: Pteromalidae) is parasitoid of Ernobius mollis in dry wood (GHAHARI et al., 2010).

Tribe Xestobiini White, 1982

Genus Hyperisus Mulsant \& Rey, 1863

\section{Hyperisus plumbeum (Illiger, 1801)}

Byrrhus coerulescens Geoffroy, 1785; Anobium thoracicum P. Rossi, 1790; Anobium politum Duftschmid, 1825; Anobium erythropum Stephens, 1830; Anobium aeneicolle Bach, 1852; Xestobium subaeneum Reitter, 1897; Xestobium syriacum Pic, 1897; Xestobium ernobiformis Reitter, 1901; Xestobium variabile Pic, 1912; Xestobium subaeneum var. circassicum Pic, 1922; Xestobium plumbeum var. rufonotatum Pic, 1923; Xestobium plumbeum var. bicoloripenne Pic, 1933; Xestobium graecum Mařan, 1941.

EXAMINED MATERIAL - Golestan province, Gorgan, $36^{\circ} 50^{\prime} \mathrm{N} 54^{\circ} 30^{\prime} \mathrm{E}, 2$ ex, 1.V.1970, Natural Sciences Museum of Barcelona, Spain (MCNB). New record for Iran.

General distribution: Armenia, Austria, Belarus,
Belgium, Bosnia-Herzegovina, Bulgaria, Croatia, Czech Republic, Cyprus, Denmark, France, Georgia, Germany, Great Britain, Greece, Hungary, Italy, Macedonia, Montenegro, Netherlands, Poland, Romania, Russia, Slovakia, Serbia, Slovenia, Spain, Syria, Switzerland, Turkey, Ukraine (ZAHRADNIK, 2007).

Hosts - It usually develops in Fagus sylvatica (Fagaceae), although it also quoted from resinous plants (ESPAÑOL, 1992).

Genus Xestobium Motschulsky, 1845

Xestobium subincanum Reitter, 1878

Xestobium circassicum Reitter, 1890.

DisTRIBUTION IN IRAN - West Azarbaijan (SAMIN et al., 2015)

General distribution - Armenia, Azerbaijan, Georgia, Iran, Italy, Russia, Turkey, Ukraine (ZAHRADNIK, 2007; SAMIN et al., 2015).

\section{Subfamily Eucradinae LeConte, 1861}

Tribe Hedobiini White, 1982

Genus Hedobia Dejean, 1821

Hedobia unicolor Pic, 1897

DistRIBUTION IN IRAN - Mazandaran (ESPAÑOL, 1977), Iran (no locality cited) (ZAHRADNIK, 2007).

General distribution - Armenia, Azerbaijan, Georgia, Iran, Russia, Saudi Arabia, Turkey (EsPAÑoL, 1977; ZAHRADNIK, 2007).

Genus Ptinomorphus Mulsant \& Rey, 1868

Ptinomorphus regalis (Duftschmid, 1825)

Hedobia regalis var. circassica Pic, 1896; Hedobia belia Reitter, 1898; Ptinomorphus regalis var. aureopilosus Pic, 1901; Hedobia regalis var. satanula Reitter, 1901; Hedobia regalis var. dispersa Pic, 1930.

DistRIBUTION IN IRAN - Iran (no locality cited) (ZAHRADNIK, 2007).

General distribution - Austria, Azerbaijan, BosniaHerzegovina, Croatia, Czech Republic, France, Georgia, Germany, Greece, Hungary, Iran, Italy, Netherlands, Poland, Romania, Russia, Slovakia, Spain, Switzerland, Turkey, Ukraine (ZAHRADNIK, 2007).

Hosts - It develops in the wood of: oak, blackthorn, hawthorn, hazel tree, etc. (ESPAÑOL, 1992).

\section{Subfamily Gibbiinae Mulsant \& Rey, 1868 Tribe Gibbiini Mulsant \& Rey, 1868 Genus Gibbium Scopoli, 1777}

Gibbium aequinoctiale Boieldieu, 1854 Gibbium chevrolati Boieldieu, 1854; Gibbium aegyptiacum Pic, 1894; Gibbium einsteini Bellés, 1980.

DISTRIBUTION IN IRAN - Northern Iran (no locality cited) (BELLÉs, 1985), Iran (no locality cited) (BOROWSKI, 2007).

General Distribution - Cosmopolitan species (BOROWSKI, 2007).

Gibbium boieldieui Levrat, 1857

Gibbium laevigena Reitter, 1884.

Distribution IN IRAN - Fars, Tehran (Bellés 1985), 
widely distributed (FARAHBAKHSH, 1961; MODARRES AWAL, 1997), Iran (no locality cited) (BOROWSKI, 2007).

General distribution - Armenia, Azerbaijan, Georgia, Greece, Iran, Russia, Turkey, Ukraine (BoROwSKI, 2007).

HOSTS - Barley, wheat and other cereals (FARAHBAKHSH, 1961; MOdARRES AWAL, 1997).

Gibbium psylloides (Czenpinski, 1778)

Ptinus scotias Fabricius, 1781; Ptinus seminulum Schrank, 1781; Bruchus apterus Geoffroy, 1785; Gibbium longicorne Reitter, 1884

DistribUTION IN IRAN - Largely distributed (FArahbaKhsh, 1961; Modarres Awal, 1997), Iran (no locality cited) (BELLÉS, 1985; BOROWSKI 2007).

General distribution - Albania, Algeria, Armenia, Austria, Azerbaijan, Belgium, Bosnia- Herzegovina, Bulgaria, China (Introduced), Croatia, Cyprus, Czech Republic, Denmark (Introduced), Egypt, Finland (Introduced), France, Great Britain, Germany, Georgia, Greece, Hungary, Iran, Iraq, Ireland (Introduced), Israel, Italy, Japan (Introduced), Lebanon, Libya, Malta, Macedonia, Moldavia, Montenegro, Morocco, Netherlands, Poland, Portugal (Madeira Archipelago), Russia (Introduced), Saudi Arabia, Serbia, Slovakia, Slovenia, South Korea (Introduced), Spain, Swede (Introduced), Switzerland, Syria, Taiwan (Introduced), Tunisia, Turkey, Ukraine (BoROWSKI, 2007).

Hosts - Wool, paper, animal and plant collections, cereals and seeds (in granary) (FARAHBAKHSH, 1961; MODARRES AWAL, 1997).

\section{Subfamily Ptilininae Shuckard, 1840 \\ Tribe Ptilinini Shuckard, 1840 \\ Genus Plumilus White, 1974}

Plumilus grandicollis (Ménétries, 1832)

Distribution IN IRAN - Iran (no locality cited) (ZAHRADNIK, 2007).

General Distribution - Armenia, Azerbaijan, Georgia, Germany (introduced), Iran, Israel, Romania, Russia, Turkmenistan, Turkey (ZAHRADNIK, 2007).

\section{Genus Ptilinus Geoffroy, 1762}

Ptilinus asiaticus Toskina, 1995

Distribution IN IRAN - Iran (no locality cited) (ZAHRADNIK, 2007).

GENERAL DISTRIBUTION - Iran, Turkmenistan (Ashkhabad, type locality) (TOSKINA, 1995; ZAHRADNIK, 2007).

Ptilinus fuscus (Geoffroy, 1785)

Ptilinus flabelicornis Le Peletier de Saint Fargeau \& Audinet-Serville, 1825; Ptilinus costatus Gyllenhal, 1827; Ptilinus flavescens (Geoffroy): Laporte de Castelnau, 1840 (non Geoffroy, 1785); Ptilinus aspericollis Mulsant \& Rey, 1853 (non Ménétries, 1832); Ptilinus asperulus Gemminger, 1870; Ptilinus mulsanti Marseul, 1886.

DisTRIBUTION IN IRAN - Golestan (SAMIN et al., 2016).

General Distribution - Afghanistan, Algeria, Austria, Belarus, Belgium, Bulgaria, China, Czech Republic, Estonia, Finland, France, Germany, Greece, Hungary, Iran, Italy, Japan, Kazakhstan, Macedonia, Netherlands, Norway, Poland, Romania, Russia, Slovakia, Spain, Sweden, Switzerland, Tajikistan, Turkey, Turkmenistan, Ukraine, Uzbekistan (ZAHRADNIK, 2007; SAMIN et al., 2016).
HosTs - Species sporadically in anthropogenic habitats. In nature it attacks branches and trunks of: beech, oak, poplar, etc. (ESPAÑOL, 1992).

Ptilinus iranicus Toskina, 1995

DISTRIBUTION IN IRAN - Tehran (TOSKINA, 1995), Iran (no locality cited) (ZAHRADNIK, 2007).

General Distribution - Known only from the type locality (Iran).

Ptilinus pectinicornis (Linnaeus, 1758)

Ptilinus cylindricus O.F. Müller, 1776; Bostrichus pectinatus Laicharting, 1781; Ptinus serraticornis Marsham, 1802 (non Thunberg, 1784); Ptilinus aspericollis Ménétries, 1832; Xiletinus discolor Faldermann, 1839; Ptilinus impressifrons Küster, 1847.

DistRIBUTION IN IRAN - Razavi Khorasan (SAMIN et al., 2016).

General Distribution - Armenia, Austria, Azerbaijan, Belarus, Bosnia-Herzegovina, Bulgaria, China, Croatia, Czech Republic, Denmark, France, Georgia, Germany, Hungary, India, Iran, Ireland, Italy, Japan, Latvia, Lithuania, Montenegro, Morocco, Netherlands, Norway, Poland, Portugal, Romania, Russia, Serbia, Slovakia, Spain, Sweden, Switzerland, Syria, Turkey, Turkmenistan, Ukraine (ZAHRADNIK, 2007; SAMIN et al., 2016).

HOSTS - Species sporadically of anthropogenic habitats. In nature attacks branches and trunks of: beech, oak, poplar, etc. (ESPAÑOL, 1992).

\section{Subfamily Ptininae Latreille, 1802 \\ Tribe Ptinini Latreille, 1802}

Genus Dignomus Wollaston, 1862

Dignomus mesopotamicus (Pic, 1894)

Distribution IN IRAN - Iran (no locality cited) (BELLÉS, 1996, 2009; BOROWSKI, 2007).

GeNerAl DisTRIBUTION - Iran, Iraq, Saudi Arabia, Yemen (BOROWSKI, 2007).

\section{Genus Niptus Boieldieu, 1856}

Niptus hololeucus (Faldermann, 1835)

Niptus brevesetosus Pic, 1956.

Distribution IN IRAN - Fars (DASHAN et al., 2014), generally distributed (FARAHBAKHSH, 1961; MODARRES AWAL, 1997), Iran (no locality cited) (BOROWSKI, 2007).

GENERAL DISTRIBUTION - It is considered a cosmopolitan species. Afghanistan, Albania, Algeria, Armenia, Austria, Belgium, Bosnia-Herzegovina, Bulgaria, Belarus, China, Croatia, Cyprus, Czech Republic, Denmark, Egypt, Estonia, Finland, France, Georgia, Germany, Great Britain, Greece, Hong Kong, Hungary, Iceland (Introduced), India, Ireland, Iran, Iraq, Israel, Italy, Japan, Jordan, Kazakhstan, Kuwait, Kyrgyzstan, Latvia, Lebanon, Liechtenstein, Lithuania, Libya, Luxembourg, Malta, Macedonia, Moldavia, Mongolia, Montenegro, Morocco, Nepal, Netherlands, North Korea, Norway, Pakistan, Poland, Portugal (Azores), Russia, Serbia, Slovakia, Slovenia, South Korea, Spain (Canary Islands), Syria, Sweden, Switzerland, Tunisia, Tajikistan, Turkey, Turkmenistan, Ukraine, Uzbekistan (BOROWSKI, 2007).

Hosts - Wool, skin, silk, linen, paper, flour, carpets, cotton and related products, tobacco, tea, medicinal plants, rice and other cereals, feathers, cork (in granary), and also 
beehive, wing and bee carcasses (FARAHBAKHSH, 1961; MODARRES AWAL, 1997).

\section{Genus Ptinus Linnaeus, 1767}

Ptinus (Bruchoptinus) elbrusicola A. Fleischer, 1915 Distribution IN IRAN - Tehran (Elburz Mountains) (FLEISCHER, 1915), Iran (no locality cited) (BoROWSKI, 2007).

General Distribution - Endemic to Iran (BorowsKi, 2007).

Ptinus (Gynopterus) quadrisignatus Ménétries, 1832 Ptinus sexsignatus Faldermann, 1835.

DisTRIBUTION IN IRAN - Ardabil (SAMIN et al., 2016).

GeNERAL DISTRIBUTION - Armenia, Azerbaijan, Georgia, India, Iran, Kazakhstan, Russia, Turkey, Uzbekistan (BorowsKi, 2007; SAMIN et al., 2016).

Ptinus (Ptinus) fur (Linnaeus, 1758)

Ptinus rapax DeGeer, 1774; Bruchus furunculus O. F. Müller, 1776; Ptinus pulex Goeze, 1776; Ptinus germanus Goeze, 1777; Ptinus striatus Fabricius, 1787; Ptinus longipes P. Rossi, 1792; Ptinus humeralis Say, 1835.

DISTRIBUTION IN IRAN - Northern and central provinces (FARAHBAKHSH, 1961; ModarRes Awal, 1997), Iran (no locality cited) (BOROWSKI, 2007).

General Distribution - Cosmopolitan species (BOROWSKI, 2007).

HosTs - Insect and other animal collections, wool, skin, seeds, dried fruits, old books, flour and related products (in granaries) (FARAHBAKHSH, 1961; ModARRES AwAL, 1997).

\section{Ptinus (Ptinus) latro Fabricius, 1775}

Ptinus testaceus A. G. Olivier, 1790; Ptinus clavipes Panzer, 1805; Ptinus brunneus Duftschmid, 1825; Ptinus hirtellus Sturm, 1837; Ptinus rufus P. H. Lucas, 1846; Ptinus hirticollis P. H. Lucas, 1846; Ptinus advena Wollaston, 1854; Ptinus lucasii Boieldieu, 1856; Ptinus corticinus Rottenberg, 1871; Ptinus obsoletus Baudi di Selve, 1874; Ptinus letourneuxi Pic, 1894; Ptinus brevipennis Pic, 1896; Ptinus furoides Escalera, 1914; Ptinus pueli Pic, 1936; Ptinus densepubens Pic, 1950; Ptinus mobilis Moore, 1957; Ptinus moorei Iablokoff-Khnzorian \& Karapetyan, 1991.

Distribution IN IRAN - Widely distributed (FARAHBAKHSH, 1961, MODARRES AWAL, 1997 as Ptinus brunneus Duftschmid, 1825), Iran (no locality cited) (MODARRES Awal, 1997 as Ptinus clavipes Panzer, 1805; BorowsKI, 2007).

General Distribution - Cosmopolitan species (BorowSKI, 2007).

Hosts - Animal and plant collections, wool, skins, silk, linen, paper, cotton and related products, flour, tobacco, tea, medicinal plants, old books, and dried fruits (in granaries) (Modarres Awal 1997 as P. brunneus), stored products (Farahbakhsh, 1961, Modarres Awal, 1997 as $P$. clavipes).

Ptinus (Ptinus) villiger (Reitter, 1884)

Ptinus balticus Iablokoff-Khnzorian \& Karapetyan, 1991.

DisTRIBUTION IN IRAN - Northern Khorasan (SAMIN et al., 2015).

General distribution - Austria, Azerbaijan, Belarus, Bosnia-Herzegovina, Bulgaria, Croatia, Czech Republic, Denmark, Estonia, Finland, Georgia, Germany, Great Britain (Introduced), Greece, Hungary, Iran, Kazakhstan,
Latvia, Lithuania, Moldavia, Montenegro, Norway, Poland, Romania, Russia, Serbia, Slovakia, Slovenia, Sweden, Turkmenistan, Ukraine; Nearctic Region (Introduced) (BorOwSKI, 2007; SAMIN et al., 2015).

Ptinus (Tectoptinus) tectus Boieldieu, 1856 Ptinus pilosus White \& Butler, 1846; Ptinus ocellus Brown, 1929.

DisTRIBUTION IN IRAN - Iran (no locality cited) (BOROWSKI, 2007).

General distribution - Cosmopolitan species (BOROWSKI, 2007).

Hosts - The species is considered as a pest in museums (Pinniger 2001). It is recorded from at least 55 museums and historic houses in the United Kingdom (PInNiger, 2012).

Subfamily Xyletininae Gistel, 1856

Tribe Lasiodermini White, 1982

Genus Lasioderma Stephens, 1835

Lasioderma costulatum Schilsky, 1899

DistriBUTION IN IRAN - Kerman (SAMIN et al., 2016).

General Distribution - Armenia, Azerbaijan, Iran, Kazakhstan, Mongolia, Tajikistan, Turkmenistan, Uzbekistan (ZAHRADNIK, 2007; SAMIN et al., 2016).

Lasioderma kiesenwetteri Schilsky, 1899

DistribuTION IN IRAN - Semnan (SAMIN et al., 2016).

General distribution - Armenia, Azerbaijan, Croatia, Czech Republic, France, Georgia, Greece, Iran, Israel, Italy, Kazakhstan, Russia, Spain, Syria, Turkey, Turkmenistan, Ukraine, Uzbekistan (ZAHRADNIK, 2007; SAMIN et al., 2016).

Lasioderma obscurum (Solsky, 1868)

Distribution IN IRAN - Iran (no locality cited) (ZAHRADNIK, 2007).

General distribution - Austria, Azerbaijan, BosniaHerzegovina, Bulgaria, Croatia, France, Georgia, Greece, Hungary, Iran, Italy, Kazakhstan, Mongolia, Montenegro, Romania, Russia, Serbia, Slovakia, Turkey, Ukraine (ZAHRADNIK, 2007).

Lasioderma redtenbacheri redtenbacheri (Bach, 1852) Xyletinus cyphonoides Moravitz, 1861; Pseudochina fulvescens Mulsant \& Rey, 1864; Lasioderma redtenbacheri var. caucasicum Pic, 1904.

DISTRIBUTION IN IRAN - Kermanshah (SAMIN et al., 2015).

General distribution - Armenia, Austria, Azerbaijan, Belgium, Bulgaria, Croatia, Cyprus, Czech Republic, Egypt, France, Georgia, Germany, Greece, Hungary, Iran, Iraq, Israel, Italy, Kazakhstan, Lebanon, Mongolia, Netherlands, Poland, Portugal, Romania, Russia, Slovakia, Spain, Switzerland, Syria, Tunisia, Turkey, Turkmenistan, Ukranie, Uzbekistan (ZAHRADNIK, 2007; SAMIN et al., 2015).

Hosts - It develops in dry chapters of Asteraceae (Centaurea, Carduus, etc.) and also in stems of Umbelliferae (Ferula) (ESPAÑOL, 1992).

Lasioderma serricorne (Fabricius, 1792)

Ptilinus testaceus Duftschmid, 1825; Lasioderma testaceum Stephens, 1835; Lasioderma castaneum Melsheimer, 1846; Xyletinus brevis Wollaston, 1861.

Distribution IN IRAN - Ardabil (GHAHARI \& LIM, 2012), Golestan (Khormali et al., 2002; MARouf et al., 2009), 
Kordestan (AllahVAISI, 2013), Mazandaran (MAROUF et al., 2009), largely distributed (MODARRES AwAL, 1997).

GENERAL DISTRIBUTION - Cosmopolitan species (EsPaÑol, 1992; ZAHRADNIK, 2007).

HOSTS - Tobacco products especially cigarettes and dried leaves and materials used in stuffing furniture, seeds and other dried plant products especially those used as drugs, black and red pepper (in granaries, tobacco factories and cigar stores) (MODARRES AwAL, 1997).

Comments - Cephalonomia gallicola (Ashmead, 1887) (Hymenoptera: Bethylidae) is a parasitoid of this species (GHAHARI \& LIM, 2012).

Tribe Xyletinini Gistel, 1856

Genus Xyletinus Latreille, 1809

Xyletinus (Xeronthobius) bucephaloides Reitter, 1901

DISTRIBUTION IN IRAN - Iran (no locality cited)

(ZAHRADNIK, 2007).

General Distribution - Cyprus, Egypt, Iran, Libya, Oman, Tunisia, Turkey (ZAHRADNIK, 2007).

Xyletinus (Xeronthobius) pallens (Germar, 1824)

Xyletinus pallidus Laporte, 1840.

DISTRIBUTION IN IRAN - Generally distributed (MODARRES AwAL, 1997), Iran (no locality cited) (ZAHRADNIK, 2007).

General Distribution - Azerbaijan, Iran, Kazakhstan,

Pakistan, Russia, Turkey, Turkmenistan, Ukraine, Uzbekistan (ZAHRADNIK, 2007).

HOSTS - Tobacco and related products (MODARRES AwAL, 1997).

Xyletinus (Xyletinus) iranicus Toskina, 2006

Distribution IN IRAN - Tehran (Naraz bei Abu Ask, Elbursgeb) (TOSKINA, 2006).

GENERAL DISTRIBUTION - Known only from the type locality (Iran).

Xyletinus (Xyletinus) laticollis (Duftschmid, 1825) Xyletinus flavipes Laporte de Castelnau, 1840; Xyletinus holosericius Dufour, 1854; Xyletinus oblongulus Mulsant \& Rey, 1864; Xyletinus flavipes var. fulvicollis Reitter, 1890; Xyletinus laticollis var. simplex Rey, 1892; Xyletinus flavipes var. flavicornis Rey, 1892; Xyletinus fulvicollis var. rufescens Schilsky, 1899; Xyletinus kocheri Pic, 1952; Xyletinus fulvicollis var. robustus Pic, 1924.

DISTRIBUTION IN IRAN - Iran (no locality cited) (ZAHRADNIK, 2007).

General distribution - Armenia, Austria, Azerbaijan, Belarus, Bosnia-Herzegovina, Bulgaria, Croatia, Czech Republic, Denmark, Egypt, Estonia, France, Germany, Greece, Hungary, Iran, Israel, Italy, Jordan, Kazakhstan, Latvia, Lebanon, Lithuania, Libya, Montenegro, Morocco, Netherlands, Norway, Poland, Portugal, Romania, Russia, Serbia, Slovakia, Spain, Syria, Sweden, Switzerland, Tajikistan, Turkey, Ukraine (Zahradnik, 2007).

HOSTS - It develops in dry droppings of sheeps and cows (ESPAÑOL, 1992).

Xyletinus (Xyletinus) sareptanus Kiesenwetter, 1877 DistrIBUTION IN IRAN - Golestan (SAMIN et al., 2016). General Distribution - Armenia, Azerbaijan, Bulgaria, Croatia, France, Georgia, Greece, Hungary, Iran, Kazakhstan, Kyrgyzstan, Macedonia, Russia, Turkey, Turkmenistan, Ukraine, Uzbekistan (ZAHRADNIK, 2007; SAMIN et al., 2015).

\section{INCERTAE SEDIS}

Abai (1984) and Modarres Awal (1997) cited Anobium denticolae Panzer (year of description is missing) from Iran (northern and central provinces) as the pest of dried wood of pine and poplar. A. denticolae does not occur in Iran. They most probably refer to Anobium denticolle Creutzer in Panzer, 1796, currently Hadrobregmus denticollis, although the genus Hadrobregmus has European distribution and is not present in Asia. So it should be a species belonging to the genus Hemicoelus, the only related genus recorded from Iran. Therefore we do not consider $A$. denticolae as part of the fauna of Iran.

\section{DISCUSSION}

Recording of 43 species of Ptinidae from Iran indicates that this family has a high species diversity in Iran. Among the 8 subfamilies reported from Iran Anobiinae with 10 species of 7 genera and Xyletininae with 10 species of 2 genera are more diverse than the other subfamilies. Two species, Ernobius iranicus and Ptinus (Bruchoptinus) elbrusicola are endemic to Iran.

Ptinids (especially Anobiinae) have several parasitoids in various insect taxa, especially subfamily Xoridinae (Hymenoptera: Ichneumonidae) (KASPARYAN, 1981), Eulophidae, Eupelmidae, Pteromalidae (Hymenoptera: Chalcidoidea) (NoYes, 2015) and Bracon spp. (Hymenoptera: Braconinae) (Yu et al., 2012). Among the Bracon species quoted as parasitoids of Anobiinae by ŽIKIĆ et al. (2012), two species [Bracon (Glabrobracon) pineti Thomson, 1892 and $B$. (Habrobracon) variegator (SPINOLA, 1808)] have been recorded from Iran by GADALLAH \& GHAHARI (2015). New findings of parasitoids from different regions of Iran can be expected in the future.

\section{ACKNOWLEDGEMETS}

Sincere gratitude to Berta Caballero, Glòria Masó (curators of the collection of arthropods, the Natural Sciences Museum of Barcelona) and N. Samin (Islamic Azad University), for the facilities provided for the consultation of the specimens, and anonymous reviewers for insightful comments. The research was supported by the Islamic Azad University (Young Researchers and Elites Club, Science and Research Branch), and by the University of Barcelona.

\section{REFERENCES}

ABai M., 1984 - List of Pests of forest trees \& shrubs of Iran. Plant Pests and Diseases Research Institute. Ministry of Agriculture and Rural Development Agricultural and Natural Resources Research Organization. Tehran. Iran, $147 \mathrm{pp}$.

AbD-Rabou S., Ghahari H., Huang J., BoučEK Z., 2005 New records of aphelinid and pteromalid wasps (Hymenoptera: Chalcidoidea: Aphelinidae, Pteromalidae) from Iran. - Egypt. J. Agric. Res., 83 (4): 1619-1623.

Allahvaisi S., 2013 - Controlling Lasioderma serricorne $F$. (Col.: Anobiidae) by Fumigation and Packaging. - World Appl. Sci. J., 28 (12): 1983-1988.

ArAngo R.A., Young D.K., 2012 - Death-watch and spider beetles of Wisconsin - Coleoptera: Ptinidae. General Technical Report FPL-GTR-209. Madison, WI: 
U.S. - Department of Agriculture, Forest Service, Forest Products Laboratory, $158 \mathrm{pp}$.

Bartolozzi L., Ghahari H., Sprecher-Uebersax E., Zilioli M., 2014 - A checklist of stag beetles (Coleoptera: Scarabaeoidea: Lucanidae) from Iran. Zootaxa, 3887 (3): 422-436.

Beaver R.A., Ghahari H., Sanguansub S., 2016 - An annotated checklist of Platypodinae and Scolytinae (Coleoptera: Curculionidae) from Iran. - Zootaxa, 4098 (3): 401-441.

BELlÉs X., 1985 - Sistemática, filogenia y biogeografía de la subfamilia Gibbiinae (Coleoptera, Ptinidae). - Treballs del Museu de Zoologia. Vol. 3; Museu de Zoologia. Ajuntament de Barcelona. Barcelona, 94 pp.

Bellés X., 1996 - El género Dignomus Wollaston (Coleoptera, Ptinidae). - Boll. Soc. d'Hist. Nat. Balears, 39: 209-228.

BELlÉS X., 2009 - Spider beetles (Coleoptera, Ptinidae) from the Socotra Archipelago. - Fauna of Arabia, 24: 145-154.

Borowski J., 2007 - Gibbiinae and Ptininae. In: Löbl, I. \& Smetana, A. (eds), Catalogue of Palaearctic Coleoptera. Vol. 4, Elateroidea, Derodontoidea, Bostrichoidea, Lymexyloidea, Cleroidea, Cucujoidea. - Apollo Books, Stenstrup, Denmark, pp. 328-339.

Bunalski M., Samin N., Ghahari H., Hawkeswood T.J., 2014 - Contributions to the knowledge the scarab beetles of Golestan province, Northern Iran with checklist of Iranian Scarabaeoidea (Coleoptera). - Polish J. Entomol., 83: 141-170.

Dashan M., SAdeghi S., BaKhshi Y., MaleK-Hosseini M.J., 2014 - First record and redescription of Niptus hololeucus (Faldermann, 1835) from Kangohar Cave (Coleoptera: Ptinidae). - Iran. J. Animal Biosyst. (IJAB), 10 (2): 81-85.

EsPaÑol F., 1963 - Notas sobre Anóbidos. 9. Contribución al conocimiento de los Gastrallus del África tropical. 10. Un nuevo género y especie de Dorcatominae del África tropical. - Revue de Zool. et Botanique d'Afrique, 67 (34): $189-202$.

Español F., 1977 - Sobre algunos Anobiidae (Col.) de Turquía recogidos por el Dr. W. Wittmer (Nota 78). Mediterranea, 2: 5-11.

Español F., 1992 - Coleoptera, Anobiidae. In: Fauna Ibérica, vol. 2, Ramos M.A. et al. (Eds.). - Museo Nacional de Ciencias Naturales. CSIC. Madrid, 195 pp.

FANTI F., GHAHARI H., 2016 - A checklist of the soldier beetles (Coleoptera: Elateroidea: Cantharidae) of Iran. Zootaxa, 4196 (4): 529-551.

FARAHBAKHSH Gh., 1961 - Bostrychidae (Coleoptera). In: Farahbakhsh Gh. (editor). A checklist of economically important insects and other enemies of plants and agricultural products in Iran. Tehran, Iran: Department of Plant Protection, Ministry of Agriculture Publication, pp. 69-70.

Fleischer A., 1915 - Ein neuer Tachys und ein neuer Ptinus vom Elburs in Nordpersien (Col.). - Entomol. Mitteilungen, 4: 130-131.

Gadallah N.S., Ghahari H., 2015 - An annotated catalogue of the Iranian Braconinae (Hymenoptera: Braconidae). - Entomofauna, 36: 121-176.

Ghahari H., AbD-Rabou S., SAKenin H., Hedqvist K.J., Ostovan H., 2010 - A contribution to some Chalcidoidea wasps (Hymenoptera) from Iran. - J. Biol. Control, 24 (1): $17-21$

Ghahari H., Lim J., 2012 - A checklist on Iranian Bethylidae (Hymenoptera: Chrysidoidea). - Linzer biol. Beiträge, 44 (1): 1071-1077.
GHAHARI H., HÁva J., 2015 - An annotated checklist of the Iranian carrion beetles (Coleoptera: Staphylinoidea: Silphidae). - Linzer biol. Beiträge, 47 (2): 1501-1511.

GHAhari H., VAZQUEZ X.A., KuBISZ D., 2017 - Annotated checklist of the false blister beetles (Coleoptera: Tenebrionoidea: Oedemeridae) from Iran. - Zootaxa, 4237 (2): 321-334.

Ghahari H., Volkovitsh M., Bellamy C.L., 2015 - An annotated catalogue of the Buprestidae of Iran (Coleoptera: Buprestoidea). - Zootaxa, 3984 (1): 1-141.

KASPARYAN D.R., 1981 - Opredelitel nasekomie evropeiskie casti USSR. Preponcatokrilie. Part III. - Zool. Inst. Russian Academy of Sciences, 688 pp.

KHORMALI S., TAHERI M.S., BORUMAND H., 2002 - Faunal investigation on stored pests in Gonbad and Minoodasht. - J. Entomol. \& Phytopathol. Appl., 70 (1): 13-23 [in Persian, English abstract].

Lasoń A., Ghahari H., 2013 - A checklist of the Kateretidae and Nitidulidae of Iran (Coleoptera: Cucujoidea). - Zootaxa, 3746 (1), 101-122.

Legalov A.A., Ghahari H., ArZanov Yu.G., 2010 Annotated catalogue of curculionid-beetles (Coleoptera: Anthribidae, Rhynchitidae, Attelabidae, Brentidae, Brachyceridae, Dryophthoridae and Curculionidae) of Iran. - Amurian zool. j., II (3), 191-244.

LiU L.-Y., Ghahari H., BeAVER R.A., 2016 - An annotated synopsis of the powder post beetles of Iran (Coleoptera: Bostrichoidea: Bostrichidae). - J. Insect Biodiversity, 4 (14): 1-22.

Marouf A., Shayesteh N., Rostam-Kolait S.E., 2009 Assessment of the efficacy of current recommendations for fumigation with Phosphine to control Lasioderma serricorne F. (Col., Anobiidae) in tobacco storages. - J. Entomol. Res., 1 (3): 249-259.

MirutenKo V., GHAHARI H., 2016 - An annotated checklist of Malachiidae (Coleoptera: Cleroidea) from Iran. Zootaxa, 4162 (2): 331-346.

Modarres Awal M., 1997 - Anobiidae, p. 128; Ptinidae, pp. 185-186. In: Modarres Awal, M. (ed.), List of agricultural pests and their natural enemies in Iran. Ferdowsi University Press, $429 \mathrm{pp}$.

Moseneagu M., 2012 - The preservation of cultural heritage damaged by anobiids (Insecta, Coleoptera, Anobiidae). - Academy of Romanian Scientists, Ann. Series Biol. Sci., 1 (2): 32-65.

NovÁK V., GHAHARI H., 2015 - A checklist of comb-clawed beetles (Coleoptera: Tenebrionidae: Alleculinae) from Iran. - Zootaxa, 4027 (1): 101-116.

Noyes J.S., 2015 - Universal Chalcidoidea Database. Natural History Museum, London. http:/www.nhm. ac.uk/jdsml/research-curation/projects/chalcidoids/ indexValidName.dsml

Otero J.C., Ghahari H., ANGelini F., 2017 - Contribution to the knowledge of cryptophagids (Coleoptera: Cryptophagidae) from Iran. - Redia, 100: 45-51; http://dx.doi.org/10.19263/REDIA-100.17.05

Pinniger D., 2001 - Pest Management in Museums, Archives and Historic Houses. - Archetype Publications. London, $124 \mathrm{pp}$.

Pinniger D., 2012 - Managing pests in paper-based collections. The Preservation Advisory Centre. - The British Library. London, $17 \mathrm{pp}$.

Platia G., Ghahari H., 2016 - An annotated checklist of click-beetles (Coleoptera, Elateridae) from Iran. Zootaxa, 4137 (2): 239-275.

SAmin N., JędryczKowski, W.B., Galini, N., SAKenin, H., NADERIAN, H., 2015 - A faunistic study on some families 
of Coleoptera from Iran. - Arquivos Entomol., 14: 253260.

SAmin N., HÁva J., KuBisz D., 2016 - A contribution to the knowledge of some families of Coleoptera (Insecta) from Iran. - Arquivos Entomol., 15: 29-38.

Thomas M.C., Ghahari H., 2016 - Checklists of Cucujidae, Laemophloeidae, and Silvanidae (Coleoptera: Cucujoidea) from Iran. - Insecta Mundi, 0498: 1-12.

Toskina I.N., 1995 - Wood-borers of the genus Ptilinus (Coleoptera Anobiidae) of Russi and adjacent countries. Russian Entomol. J., 4 (1-4): 15-34.

Toskina I.N., 2006 - New Palaearctic species of the genus Xyletinus Latreille, 1809 (Coleoptera: Anobiidae). Zoosys. Rossica, 14 (2): 223-260.

VIÑolas A., 2013a - Els Dorcatominae de la península Ibèrica i illes Balears. 2 a nota. El gènere Dorcatoma Herbst, 1792 (Coleoptera: Ptinidae). - Orsis, 27: 7-28.

VIÑolas A., 2013b - Els Dorcatominae de la península Ibèrica i illes Balears. 3 a nota. El gènere Stagetus Wollaston, 1861, amb la descripció de $\mathrm{S}$. confusus $n . s p$. (Coleoptera: Ptinidae). - Orsis, 27: 95-121.

VIÑOLAS A., 2013c - Els Mesocoelopodinae de la Peninsula Ibèrica $i$ les illes Balears. Els gèneres Mesocoelopus Jacquelin du Val, 1860, Mesothes Mulsant \& Rey, $1864 i$ Rhamna Peyerimhoff, 1913 (Coleoptera: Ptinidae). Butll. Inst. Catalana d'Hist. Nat., 77: 105-116.

VIÑolas A., 2013d - Els Dorcatominae de la península Ibèrica $i$ de les illes Balears. Ia nota. Els gèneres Caenocara C.G. Thomson, 1859, Calymmaderus Solier, 1849 i Mizodorcatoma Hayashi, 1955 (Coleoptera: Ptinidae). - Butll. Inst. Catalana d'Hist. Nat., 77: 117-132.
ViÑOlAS A., MASÓ G., 2007 - Revisión del género Stagetus Wollaston, 1861, en el África continental: descripción de n. sp. y nuevas citas para la Península Ibérica. Falsostagetus $n$. gen. y n. sp. para el África austral (Coleoptera: Anobiidae: Dorcatominae). - Butll. Inst. Catalana d'Hist. Nat., 74 (2006): 37-80.

Yu D.S., Achterberg C. van, Horstmann K., 2012 World Ichneumonoidea 2005. Taxonomy, biology, morphology and distribution [Braconidae]. - Taxapad 2006 (Scientific names for information management) Interactive electronical catalogue on DVD/CD-ROM. Vancouver.

ZAHRADNík P., 2007 - Ptinidae (except Gibbiinae and Ptininae, pp. 339-362. In: Löbl, I. \& Smetana, A. (eds), Catalogue of Palaearctic Coleoptera. Vol. 4, Elateroidea, Derodontoidea, Bostrichoidea, Lymexyloidea, Cleroidea, Cucujoidea. - Apollo Books, Stenstrup, Denmark, 935 pp.

ZAHRADNIK P., 2016 - A new species of the genus Ernobius C.G. Thomson, 1859 from Iran (Coleoptera: Bostrichoidea: Ptinidae). - Studies and Reports, Taxonomical Series, 12 (2): 495-498.

ZAPPI I., GHAHARI H., 2016 - A checklist of the Cleridae of Iran with new data (Coleoptera: Cleroidea). - Zootaxa, 4147 (4): 403-420.

Zehzad B., Kiabi B.H., Madjnoonian H., 2002 - The natural areas and landscape of Iran: an overview. - Zool. Middle East, 26: 7-10.

ŽIrić V., STANKović S.S., Ilić M., 2012 - Checklist of the genus Bracon (Hymenoptera: Braconidae) in Serbia. Biologica Nyssana, 3 (1): 21-29. 
148 - Blank Page 